< TI > Team Processes in Canadian Primary Healthcare: A Realist Review

$<$ AU1>Maura MacPhee, Geoffrey Wong, Ruth Abrams and Katelyn Merrett

$<$ H1 $>$ Abstract

$<$ TX1 > To provide effective, comprehensive care to increasingly complex patients in Canadian communities, healthcare providers are shifting from solo providers of primary care to interprofessional, team-based primary healthcare services. Team-based care is considered as one of the most effective means of caring for complex patients, including frail elders and individuals with chronic illness, mental health issues and addictions. Team-based care relies on effective team processes, the social or relational processes that enhance team collaboration and decision making. This realist review will highlight the team processes associated with high-performing teams and provide team development and sustainment strategies for providers and healthcare decision makers.

\title{
[END ABSTRACT]
}

\section{$<$ H1 $>$ Background}

$<$ TX1>In 2003, Canada's first ministers endorsed team-based care delivery as a major tenet of primary healthcare reform (Health Council of Canada 2005). Reform was introduced to more effectively manage increasing numbers of Canadians with chronic health conditions and social care needs (Health Council of Canada 2009). Resources were allocated through the Primary Health Care Transition Fund (Government of Canada 2007) to pilot team-based models. The Health Council of Canada (2009) defined team-based care delivery as two or more healthcare providers coordinating basic healthcare services for patients. Since the initial reform efforts, there has been a gradual national uptake of team-based primary healthcare delivery (Misfeldt et al. 2017).

$<$ TX2 $>$ Team-based care delivery is considered the most effective and efficient way of delivering services to patients with complex needs (Salas et al. 2015). Team effectiveness, however, is contingent upon how teams work together to reach shared goals. Team processes are specific social processes associated with high-performing teams, and they are the interpersonal building blocks of teamwork (LePine et al. 2008). Although an extensive body of teamwork literature 
exists across the social sciences, health and business, healthcare teams still struggle to provide quality, safe care (LePine et al. 2008)

This review provides evidence-informed guidance of how effective teams develop and sustain themselves within Canadian primary healthcare settings by asking the following questions: (1) What team processes are associated with team effectiveness in Canadian primary healthcare settings ? (2) What mechanisms and contextual factors result in team effectiveness in Canadian primary healthcare settings?

\section{$<$ H1 $>$ Method}

$<$ TX1 $>$ Team effectiveness is complex, and the evidence is multifaceted. A realist review approach can account for this complexity. Realist reviews go beyond other types of reviews to explain why programs or processes fail or succeed within specific contexts (e.g., Canadian primary healthcare settings). Realist reviews are used to develop testable context-mechanismoutcomes configurations (CMOCs). An outcome is caused by a mechanism that is activated within a certain context. Contexts usually represent "for whom" and "in what circumstances," and mechanisms address "why" and "how" with respect to intended outcomes (Berg and Nanavati 2016; Pawson 2006).

$<$ TX2>Adhering to the Realist And Meta-narrative Evidence Syntheses-Evolving Standards realist review quality standards (Wong et al. 2014), we constructed a search strategy for CINAHL and Medline Ovid using the following primary search terms: "primary health care," "Canada” and "team.” We searched the TRIP medical database using these same terms. Other inclusion criteria were English-language documents within the past 20 years. A total of 2,103 abstracts were screened by two reviewers for inclusion criteria. The final selection of 118 full- 
text documents were examined by two reviewers for sufficient rigour (Pawson 2006) and used to answer the research questions. The final full-text documents were uploaded in NVivo 12 and coded by one researcher; a second researcher performed consistency checks on $10 \%$ of the coded data. Data were coded as potential CMOCs (Abrams et al. 2018; RAMESES II Project 2017). Please see the article by MacPhee et al. (2020) for the complete review protocol with search tables and the PRISMA chart.

\section{$<$ H1 $>$ Results}

$<\mathbf{H} 2>$ What team processes are associated with team effectiveness in primary care contexts?

<TX1>Tuckman's phases of team development framed our findings (Bonebright 2010), being one of the most valid, reliable model of small group/team development. The model includes forming, storming/norming and performing phases (Figure 1). Figure 1 displays key concepts, derived from the coded review documents, that represent team processes along the team development trajectory. Although the figure depicts a linear process, in reality, team members may enter, exit or be at different points in time along this pathway.

[CATCH FIGURE 1; CAPTION:]

$<$ CA $>$ FIGURE 1. Program theory for team processes in Canadian primary healthcare settings

\section{$<$ H2 $>$ What mechanisms and contextual factors result in team effectiveness?}

$<$ TX1 $>$ The key team processes for each team development phase are in Table 1 with corresponding, numbered CMOCs. Factors that function as a mechanism in one CMOC can function as context or outcome within other CMOCs. In other words, factors are not "fixed.” The 
following sections link Table 1 CMOCs to document evidence and to substantive theories from psychology and sociology.

[CATCH TABLE 1; CAPTION:]

$<$ CA $>$ TABLE 1. CMOCs for team processes program theory

$<$ H2>Forming

$<\mathbf{H 3}>$ CMOC 1

$<$ TX1 $>$ During the forming phase, key team processes include the establishment of social grounds rules and learning about each other's scopes of practice and professional roles (Bareil et al. 2015). The team context provides opportunities for teams to become socialized to each other, and the underlying mechanism or trigger for team socialization is perspective-taking. Perspective-taking theory (Galinsky et al. 2005) explains that when individuals gather together, they foreground similarities among themselves and they ignore or minimize differences. Perspective-taking is the social glue that increases our capacity to collaborate and coordinate more effectively over time (Todd and Galinsky 2014).

$<$ TX2 $>$ Perspective-taking is augmented during team meetings as team members discuss what they do and how they contribute to patient care (Bareil et al. 2015). Role and scope clarification are the typical foci of these meetings. Frequent, regular structured meetings are necessary to foster constructive dialogue about shared team goals and to clarify role expectations (Bareil et al. 2015; Brown and Ryan 2018; Brown et al. 2015; Chreim et al. 2010). The intended outcomes of team socialization are social bonds and trusting relationships. As stated by one medical practitioner, "People need to learn about the scope of practice and skills that different 
professionals bring to the team. It's not enough to just write it down. You have to build trusting relationships. This takes years, not months” (Collier 2011, p. 1131).

\section{$<$ H3 $>$ CMOC 2}

$<$ TX1 $>$ At the onset of team development, dedicated support (a contextual factor) may facilitate proactive planning, facilitation and, in many instances, skilled coordination depending on the number of team members and external stakeholders engaged in care delivery transformation (Bareil et al. 2015; Brault et al. 2014; Contandriopoulos et al. 2015). Team members, such as physicians, are often expected to lead team formation. When this happens in addition to practice, the hard work of socialization is often under-recognized.

$<$ TX2 $>$ With dedicated support in place, team members have greater opportunity for mutual perspective-taking, the mechanism of team socialization. The expected outcome is increased awareness of each other's roles and scopes. Some teams find an external facilitator useful at this stage, "The facilitator serves as a catalyst by stimulating clinicians' reflections about their own practices and the necessary changes” (Bareil et al. 2015, p. 289).

\section{$<\mathbf{H} 3>$ CMOC 3}

$<$ TX1 $>$ Team members with certain contextual characteristics, such as self-other awareness and reciprocity, are more likely to initiate interactions and promote closer team bonds. In one Montreal clinic setting, a newly introduced nurse practitioner (NP) "took the time to understand what the local needs were and to elaborate her role on the basis of these needs and her experience” (Chreim et al. 2010, p. 193). In another clinic when NPs were introduced to the practice, the general practitioners stated, "First we started by meeting them. Then...to learn from them what were their abilities, their limitations, and what they wanted to do as work" (Brault et 
al. 2014, p. 6). In this clinic, it took six months for the NPs to settle into their role, and up to a year for their role to be fully integrated within the team - it depends on the mechanism carefulness.

$<$ TX2>Carefulness consists of upholding social rules of civil behaviour. It reflects the importance of being heedful or careful toward our colleagues (Shain 2016; Shain and Mills 2002). For example, when we are aware of others' thoughts and actions and our influence on them, our carefulness contributes to a psychologically safe workplace (Shain 2016). In addition to Shain’s (2016) work on carefulness and psychological safety, Edmondson’s (1999) seminal work on team learning behaviour found that when teams feel psychologically safe, they share important information, they make decisions together and they learn and perform better together. Psychological safety, an outcome, represents people’s perceptions of their interpersonal risks for harm (e.g., harassment, bullying) and their willingness to contribute to the team (Edmondson and Lei 2014).

\section{$<\mathbf{H} 2>$ Storming $<$ H3 $>$ CMOC 4}

$<$ TX1 $>$ The storming phase is characterized as a time of conflict when the team tests out their roles in a new care-delivery model (Bonebright 2010). During storming, carefulness functions as a contextual factor. When team members lack carefulness toward one another, they fail to take other's perspectives. Perspective-taking continues to serve as a critical mechanism during storming. Perspective-taking is necessary to break down stereotypes, power differentials and prejudice. When team members ignore or override others' perspectives, the outcome is a widening us-them gap, fuelling conflict (Galinsky et al. 2011; Gilin et al. 2015). 


\section{$<\mathbf{H} 2>$ Norming}

$<\mathbf{H 3}>$ CMOC 5

$<$ TX1 > Safe passage from storming to norming requires investment in contextual factors such as planned time and space for ongoing team interactions (Contandriopolous et al. 2015). After initial energy and investment in team formation, further resource allocation for teamwork often wanes, and teams focus on tasks (taskwork; Griffith and Yombo 2015). Taskwork consists of patient care activities, whereas teamwork consists of goal-setting, coordination and monitoring; affect management (e.g., conflict management); and debriefing, information sharing and decision making as a team (Marks et al. 2001). There must be regular, frequent opportunities for teams to do teamwork as well as taskwork: All action without reflection can result in dysfunction. As one Ontario family health team said, "You're going to have to sit down and talk to each other about the ambiguity and uncertainty of the work [you] do. Yeah, it’s a big change.” (Goldman et al. 2010, p. e372).

$<$ TX2 $>$ Perspective-taking continues to operate as the key mechanism, and the norming outcome is deeper trust development or affective trust. According to trust formation theory, cognitive trust develops first, followed by trust forged through closer working relationships. Affective trust takes longer to develop, and it forms as team members learn whether their colleagues will back them up and support them when they need help (Webber 2008). For example, as interdisciplinary team members in Edmonton, Alberta, established new ways of thinking and working together, they “co-created new [team] norms” (Asselin et al. 2017, p. E328). The concept of shared mental models suggests that team members’ capacity to read each other’s signals and respond reflexively to each other may be a demonstration of affective trust (McComb et al. 2017). As stated from a team member in one Toronto clinic, “That rapport for the physicians to 
really...they are officially letting you into the circle of care before they trust your recommendations and feel comfortable with it. It's just [takes] time...” (Gucciardi et al. 2016, p. 6).

$<\mathbf{H} 3>$ CMOC 6

$<$ TX1 $>$ Open sharing of information and ideas and even social bonding are contextual factors associated with affective trust, the mechanism underlying the transition from a group of providers to a collective team identity, an expected outcome of norming. A quote from a Toronto family health clinic captures team identity: "The team works best with people who really care about what they're doing... We're proud to be part of this team. We want to provide excellent service, excellent care, excellent teamwork; we're all on the same page where we all have the same goals” (Brown et al. 2015, p. 197). A dietician describes their comfort with learning through other team members' perspectives: “Just collaborating at the end, having an open discussion, getting perspectives from different health care professionals is always good too..." (Asselin et al. 2016, p. 130).

\section{$<$ H2 $>$ Performing $<$ H3 $>$ COMC 7}

$<$ TX1 $>$ The mechanisms of carefulness and perspective-taking become the contextual backdrop for teams to evolve into high-performing teams via the mechanism of affective trust. An NP from a British Columbia primary healthcare clinic stated the following:

$<$ BQ $>$ When you feel you're actually being cared for as a person it's amazing how that plays into how you work ... there's some quality, some sort of sensibility. Some sort of feeling 
of connectedness that isn't created, isn't manufactured ....And it's a safe environment; it's the climate, it’s culture (Burgess and Purkis 2010, p. 302).

$<$ TX2>High-performing teams are a hallmark of the performing phase (Bonebright 2015). Expected outcomes include flexibility, adaptability and comfort with change.

$<$ TX3 $>$ Change and ambiguity are often threatening to less-developed teams; high-performing teams acknowledge the importance of improvement opportunities. Brown and Ryan (2018, p. e287) tracked the evolution of 20 family health teams in Ontario. Members of a developing team described change as challenging and threatening: "I think change is hard for everyone .... People are always scared of change.” Whereas members of an established, high-performing team described change as an inherent part of getting better as a team: "Change has been so normal here that people are just sort of used to it .... I think they [team members] embrace it because if they didn’t embrace it they wouldn’t be here.”

\section{$<$ H1 $>$ Discussion}

$<$ TX1>Seven CMOCs, constructed from a realist review of 118 documents, illustrate how key mechanisms of human behaviour are activated within specific contexts to explain the transition from forming teams to becoming high-performing teams: Each CMOC links contexts and mechanisms to intended outcomes. Because mechanisms are the drivers of people's actions within specific contexts, we will describe evidence-informed strategies from the review literature that are associated with our key mechanisms (Table 2).

[CATCH TABLE 2; CAPTION:]

$<$ CA $>$ TABLE 2. Evidence-informed strategies for key team processes mechanisms $<$ H2 > Perspective-taking strategies 
$<$ TX1 > Perspective-taking requires frequent opportunities to observe, interact and learn about one another. Team mapping is one method for discussing each other's team roles and accountabilities with respect to patient-centred care. Team mapping acts as an "ice breaker” during new team formation and orientation of new members to established teams (Price et al. 2019). Team learning can be fostered through regular ongoing communications with each other, such as formalized team meetings (Sims et al. 2015), scheduled team briefings at the start of the day with brief huddles during the day (Shunk et al. 2014) and debriefings at the end of the day (Eddy et al. 2013). These team communication strategies create the context needed to enable team perspective-taking, also described in the literature as team reflexivity (Schumtz et al. 2017). Team communications strategies are also associated with the quality/safety movement, and the global Institute for Healthcare Improvement (IHI 2019) provides a variety of online resources.

\section{$<\mathbf{H} 2>$ Carefulness}

<TX1>Carefulness underlies the development of psychological safety, and carefulness can be cultivated through relational education (Shain 2016; Shain and Mills 2002). Human attention wanders, and mindfulness training “stabilizes” attention in the present, so that others feel appreciated and listened to. Research evidence suggests that mindfulness training improves interpersonal behaviours in team relationships, such as open listening with less evaluative judgment of others (Good et al. 2016; Ruff and Mackenzie 2009). Other relational strategies to cultivate carefulness come from the field of positive psychology, a field that studies individuals’ character strengths (Gable and Haidt 2005). Reflected best self is a simple positive psychology practice of reminding individuals to stop and reflect before doing or saying anything, to consider if it is the best reflection of oneself (Roberts et al. 2005). Appreciative inquiry, another positive psychology strategy, can be used at the team level during huddles, team briefings and debriefings 
to ask the following questions: "What is working well?” "What can we do better?” "How can we best support each other?” (Richer et al. 2009).

\section{$<\mathbf{H} 2>$ Trust}

<TX1>Although interprofessional health education is widely endorsed, the majority of pregraduate health disciplines education happens in silos (Salas et al. 2009). Teams begin with groups of providers who know very little about each other's roles and scope, and trust requires knowledge of each other's capacity to carry out taskwork competently (i.e. quick trust) and to support team members as needed (i.e., affective trust). A commonly overlooked component of the forming phase is establishment of teamwork structures and processes, such as ground rules, to ensure respectful communications (Ghorob and Bodenherimer 2015) and assignment of a designated facilitator to “smooth” hierarchical relationships (Bareil et al. 2015). There are many cases of team dysfunction and power dynamics that derail forming teams (Almost et al. 2016). Other interactional strategies that support initial trust formation include formal facilitated orientation (Lessard et al. 2016) and shadow experiences among team members that provide opportunities to observe one another's work, ask questions and acquire comfort with each other's roles and accountabilities (Jain et al. 2012).

\section{$<\mathbf{H} 2>$ What Is Missing?}

$<$ TX1 $>$ Our review did not extend into the leadership literature and the importance of informal and formal leadership roles within teams. As shown in Figure 1, effective leadership is critical across the team development continuum. Brown and Ryan (2018, p. 102) described the impact of different leadership styles within Ontario family health teams. The researchers found that “progressing” teams had respected and inspirational leaders. "Stalled” teams had leaders who 
were autocratic or laissez-faire, abdicating any responsibility. Although our review documents contain some evidence to support the importance of effective leadership styles, what needs more investigation are the contexts and mechanisms associated with effective team leadership in teambased primary healthcare settings. Another issue that we did not cover through this review is funding models and the management of primary healthcare services from an economic and business perspective. The impact of funding on team processes needs further investigation (Longhurst and Cohen 2019).

\section{$<$ H2>Conclusions and Policy Implications}

$<$ TX1 $>$ Team-based care holds promise as the most effective approach to primary healthcare services delivery (Salas et al. 2008). Our realist review focused on Canadian primary healthcare settings, but we found significant evidence throughout the review process that our identified team processes may operate in other healthcare settings in Canada and in other countries. Team processes are social processes that influence team effectiveness, and these team processes require the right contextual factors to ensure that the fundamental mechanisms of perspective-taking, carefulness and trust formation happen throughout team development.

$<$ TX2 $>$ We recommend that relational education strategies, such as mindfulness training, should be in place for all healthcare professionals before graduation and postgraduation, such as continuing professional development opportunities (Konrad and Browning 2012). From a policy perspective, successful team formation requires investment in formal team orientation with trained facilitators and ongoing change management support: It is too much for healthcare providers to manage in addition to their clinical responsibilities. Policy makers must also acknowledge the importance of ongoing team training and support throughout the other development phases of the team. Initial team development efforts fail without continued 
investment in time and space to meet, interact and establish affective trust relationships - the stuff of high-performing teams.

\section{$<$ RH $>$ References}

$<$ RTX>Abrams, R., G. Wong, K. Mahtani, S. Tierney, A-M Boylan, N. Roberts et al. 2018. Understanding the Impact of Delegated Home Visiting Services Accessed via General Practice by Community-Dwelling Patients: A Realist Review Protocol. BMJ Open 8: e024876. doi:10.1136/bmjopen-2018-024876.

Almost, J., A. Wolff, A. Stewart-Pyne, L. McCormick, D. Strachan and C. D’Souza. 2016. Managing and Mitigating Conflict in Healthcare Teams: An Integrative Review. Journal of Advanced Nursing 72(7): 1490-505. doi:10.1111/jan.12903.

Asselin, J., E. Salami, A. Osunlana, A. Ogunleye, A. Cave, J. Johnson et al. 2017. Impact of the 5As Team Study on Clinical Practice in Primary Care Obesity Management: A Qualitative Study. Canadian Medical Association Journal Open 5(2): E322-29. doi:10.9778/cmajo.20160090.

Bareil, C., F. Duhamel, L. Lalonde, J. Goudreau, E. Hudon, M-T Lussier et al. 2015. Facilitating Implementation of Interprofessional Collaborative Practices into Primary Care: A Trilogy of Driving Forces. Journal of Healthcare Management 60(4): 287-300. doi:10.1097/00115514201507000-00010.

Berg, R. and J. Nanavanti. 2016. Realist Review: Current Practice and Future Prospects. Journal of Research Practice 12(1): Article R1. Retrieved <>. $<$ http://jrp.icaap.org/index.php/jrp/article/view/538/449>.

Bonebright, D. 2010. 40 Years of Storming: A Historical Review of Tuckman's Model of Small Group Development. Human Resource Development International 13(1): 111-20. doi:10.1080/13678861003589099.

Brault, I., K. Kilpatrick, D. D’Amour, D. Contandriopoulos, V. Chouinard, C. Dubois, et al. 2014. Role Clarification Processes for Better Integration of Nurse Practitioners into Primary Healthcare Teams: A Multi-Case Study. Nursing Research and Practice 2014: 170514. doi:10.1155/2014/170514.

Brown, J. and B. Ryan. 2018. Processes that Influence the Evolution of Family Health Teams. Canadian Family Physician 64(6): e283-89.

Brown, J., B. Ryan, C. Thorpe, E. Markle, B. Hutchinson and R. Glazier. 2015. Measuring Teamwork in Primary Care: Triangulation of Qualitative and Quantitative Data. Families, Systems, \& Health 33(3): 193-202. doi10.1037/fsh0000109.

Burgess, J. and M.E. Purkis. 2010. The Power and Politics of Nurse Practitioner Role Development. Nursing Inquiry 17(4): 297-308. doi:10.1111/j.1440-1800.2010.00505.x. 
Chreim, S., B. Williams, L. Janz and A. Dastmalchian. 2010. Change Agency in a Primary Health Care Context: The Case of Distributed Leadership. Healthcare Management Review 35(2): 187-99. doi:10.1097/HMR.0b013e3181c8b1f8.

Collier, R. 2011. Verdict Is Still Out on Family Health Teams. CMAJ 183(10): 1131-32. doi:10.1503/cmaj.109-3864.

Contandriopoulos, D., A. Brouselle, C. Dubois, M. Perroux M.-D. Beaulieu, I. Brault et al. 2015. A Process-Based Framework to Guide Nurse Practitioners' Integration into Primary Healthcare Teams: Results from a Logic Analysis. BMC Health Services Research 15(1): 78. doi:10.1186/s12913-015-0731-5.

de Stampa, M., I. Vedel, H. Bergman, J.-C. Novella, L. Lechowski, J. Ankri et al. 2013. Opening the Black Box of Clinical Collaboration in Integrated Care Models for Frail, Elderly Patients. The Gerontologist 53(2): 313-25. doi:10.1093/geront/gns081.

Eddy, E., S. Tannenbaum and J. Mathieu. 2013. Helping Teams to Help Themselves: Comparing Two Team-Led Debriefing Methods. Personnel Psychology 66(4): 975-1008. doi:10.1111/peps.12041.

Edmondson, A. 1999. Psychological Safety and Learning Behavior in Work Teams. Administrative Science Quarterly 44(2): 350-83. doi:10.2307/2666999.

Edmondson, A.C. and Z. Lei. 2014. Psychological Safety: The History, Renaissance, and Future of an Interpersonal Construct. Annual Review of Organizational Psychology and Organizational Behavior 1: 23-43.

Gable, S. and J. Haidt. 2005. What (and Why) Is Positive Psychology? Review of General Psychology 9(2): 103-110. doi:10.1037/1089-2680.9.2.103.

Galinsky, A., D. Gilin and W. Maddux. 2011. Using Both Your Head and Your Heart: The Role of Perspective Taking and Empathy in Resolving Social Conflict. In J. Forgas, A. Kruglanski and K. Williams, eds., The Psychology of Social Conflict and Aggression (pp. 103-18). Taylor \& Francis.

Galinsky, A., G. Ku and C. Wang. 2005. Perspective-Taking and Self-Other Overlap: Fostering Social Bonds and Facilitating Social Coordination. Group Processes and Intergroup Relations 8(2): 109-24. doi:10.1177/1368430205051060.

Ghorob, A. and T. Bodenheimer. 2015. Building Teams in Primary Care: A Practical Guide. Family Systems \& Health 33(3): 182-92. doi.org/10.1037/fsh0000120.

Gilin, D., M. Leiter and D. LeBlanc. 2015. Individual and Organizational Factors Promoting Successful Responses to Workplace Conflict. Canadian Psychology 56(3): 301-10. doi:10.1037/cap0000032.

Goldman, J., J. Meuser, J. Rogers, L. Lawrie and S, Reeves. 2010. Interprofessional Collaboration in Family Health Teams: An Ontario-Based Study. Canadian Family Physician 56: e368-74. 
Good, D., C. Lyddy, T. Glomb, J. Bono, K. Brown, M. Duffy, et al. 2016. Contemplating Mindfulness at Work: An Integrative Review. Journal of Management 42(1): 114-42. doi:10.1177/0149206315617003.

Griffith, B. and B. Yombo. 2015. Keep the Momentum Going: A Project Manager's Plan to Sustaining High Team Performance on Time-Sensitive Projects. PM World Journal 4(8): 1-12.

Government of Canada. 2007. Primary Health Care Transition Fund. Retrieved December 20, 2019. <https://www.canada.ca/en/health-canada/services/primary-health-care/primary-healthcare-transition-fund.html>.

Gucciardi, E., S. Espin, A. Morganti and L. Dorado. 2016. Exploring Interprofessional Collaboration during the Integration of Diabetes Teams into Primary Care.” BMC Family Practice 17: 12. doi:10.1186/s12875-016-0407-1.

Health Council of Canada. 2005 January. Health Care Renewal in Canada: Accelerating Change. Retrieved December 20, 2019. <https://healthcouncilcanada.ca/files/2.48Accelerating_Change_HCC_2005.pdf $>$.

Health Council of Canada. 2009, April. Teams in Action: Primary Health Care Teams for Canadians. Retrieved December 20, 2019. <https://healthcouncilcanada.ca/files/2.42teamsinaction_1.pdf $>$.

Lee, B., D. Shannon, P. Rutherford and C. Peck.. 2008. Transforming Care at the Bedside How-to Guide: Optimizing Communication and Teamwork. Institute for Healthcare Improvement (IHI). Retrieved December 23, 2019.

<http://www.ihi.org/resources/Pages/Tools/TCABHowToGuideOptimizingCommunicationTeam work.aspx $>$.

Jain, A., E. Luo, J. Yang, J. Purkiss and C. White. 2012. Implementing a Nurse-Shadowing Program for First-Year Medical Students to Improve Interprofessional Collaborations on Health Care Teams. Academic Medicine 87(9): 1292-95. doi:10.1097/ACM.0b013e31826216d0.

Konrad, S. and D. Browning. 2012. Relational Learning and Interprofessional Practice: Transforming Health Education for the 21st Century. Work 41(3): 247-51. doi:10.3233/WOR2012-1295.

LePine, J., R. Piccolo, C. Jackson, J. Mathieu and J. Saul. 2008. A Meta-analysis of Teamwork Processes: Tests of a Multidimensional Model and Relationships with Team Effectiveness Criteria. Personnel Psychology 61(2): 273-307. doi:10.1111/j.1744-6570.2008.00114.x.

Lessard, S., C. Bareil, L. LaLonde, F. Duhamel, E. Hudon, J. Goudreau et al. 2016. External Facilitators and Interprofessional Facilitation Teams: A Qualitative Study of Their Roles in Supporting Practice Change. Implementation Science 11: 97. doi:10.1186/s13012-016-0458-7.

Longhurst, A. and M. Cohen. 2019, March 1. Policy Note: The Importance of Community Health Centres in BC's Primary Care Reforms: What the Research Tells Us. Retrieved December 23, 2019. 
<https:/www.policyalternatives.ca/sites/default/files/uploads/publications/BC\%20Office/2019/03/ccpabc_march2019_chcs-in-bc.pdf>.

MacPhee, M., G. Wong, R. Abrams and K. Merrett. 2020, February 28. Team Processes in Canadian Primary HealthCare: A Realist Review Protocol. UBC Faculty Research and Publications. Retrieved March 23, 2020.

<https://open.library.ubc.ca/cIRcle/collections/facultyresearchandpublications/52383/items/1.0388742>.

Marks, M.A., J.E. Mathieu and S.J. Zaccaro. 2001. A Temporally Based Framework and Taxonomy of Team Processes. The Academy of Management Review 26(3): 356-76. doi:10.5465/amr.2001.4845785.

McComb, S.A., M. LeMaster, E.A. Henneman and K.T. Hinchey. 2017. An Evaluation of Shared Mental Models and Mutual Trust on General Medical Units: Implications for Collaboration, Teamwork, and Patient Safety. Journal of Patient Safety 13(4): 237-42. doi:10.1097/PTS.0000000000000151.

Misfeldt, R., E. Suter, S. Mallinson, O. Boakye, S. Wong and L. Nasmith. 2017. Exploring Context and the Factors Shaping Team-Based Primary Healthcare Policies in Three Canadian Provinces: A Comparative Analysis. Healthcare Policy 13(1): 74-93. doi:10.1016/j.xjep.2016.11.004.

Pawson, R. 2006. Evidence-based Policy: A Realist Perspective. SAGE Publications.

Price, M., P. Bellwood, T. Hill and S. Fletcher. 2020. Team Mapping: A Novel Method to Help Community Primary Healthcare Practices Transition to Team-Based Care. Healthcare Quarterly 22(4): 33-39. doi:10.12927/hcq.2020.26086.

Greenhalgh, T., R. Pawson, G. Wong, G. Westhorp, J. Greenhalgh, A. Manzano, et al. 2017. Retroduction in Realist Evaluation: The RAMESES II Project. Retrieved June 16, 2020. $<$ https://www.ramesesproject.org/media/RAMESES_II_Retroduction.pdf>.

Richer, M.-C., J. Ritchie and C. Marchionni. 2009. If We Can’t Do More, Let's Do It Differently! Using Appreciative Inquiry to Promote Innovative Ideas for Better Health Care Work Environments. Journal of Nursing Management 17(8): 947-55. doi:10.1111/j.13652834.2009.01022.x.

Roberts, L.M., J.E. Dutton, G.M. Spreitzer, E.D. Heaphy and R.E. Quinn. 2005. Composing the Reflected Best Self Portrait: Building Pathways for Becoming Extraordinary in Work Organizations. Academy of Management 30(4): 712-36. doi:10.5465/amr.2005.18378874.

Ruff, K.M. and E.R. Mackenzie. 2009. The Role of Mindfulness in Healthcare Reform: A Policy Paper. Explore 5(6): 313-23. doi:10.1016/j.explore.2009.10.002.

Salas, E., K. Wilson, C. Murphy, H. King and M. Salisbury. 2008. Communicating, Coordinating, and Cooperating When Lives Depend on It: Tips for Teamwork. The Joint 
Commission Journal on Quality and Patient Safety 34(6): 333-41. doi:10.1016/s15537250(08)34042-2.

Salas, E., S.A. Almeida, M. Salisbury, H. King, E.H. Lazzara, R. Lyons, et al. 2009. What Are the Critical Success Factors for Team Training in Health Care? The Joint Commission Journal on Quality and Patient Safety 35(8): 398-405. doi:10.1016/s1553-7250(09)35056-4.

Salas, E., M. Shuffler, A. Thayer, W. Bedwell and E. Lazzara. 2015. Understanding and Improving Teamwork in Organizations: A Scientifically Based Practical Guide. Human Resource Management 54(4): 599-622. doi:10.1002/hrm.21628.

Schumtz, J. and W. Eppich. 2017. Promoting Learning and Patient Care Through Shared Reflection: A Conceptual Framework for Team Reflexivity in Health Care. Academic Medicine 92(11): 1555-63. doi:10.1097/ACM.0000000000001688.

Shain, M. 2016. The Careful Workplace: Seeking Psychological Safety at Work in the Era of Canada's National Standard. Thomson Reuters.

Shain, M. and D. Mills. 2002. The Neighbour at Work. Occupational Health \& Safety Canada 18(6): 1-9.

Shunk, R., M. Dulay, C.L. Chou, S. Janson and B.C. O’Brien. 2014. Huddle-Coaching: A Dynamic Intervention for Trainees and Staff to Support Team-Based Care. Academic Medicine 89(2): 244-50. doi:10.1097/ACM.0000000000000104.

Sims, S., G. Hewitt and R. Harris. 2015. Evidence of Collaboration, Pooling of Resources, Learning and Role Blurring in Interprofessional Healthcare Teams: A Realist Synthesis. Journal of Interprofessional Care 29(1): 20-25. doi:10.3109/13561820.2014.939745.

Todd, A.R. and A.D. Galinsky. 2014. Perspective-Taking as a Strategy for Improving Intergroup Relations: Evidence, Mechanisms and Qualifications. Social and Personality Psychology 8(7): 374-87. doi:10.1111/spc3.12116.

Webber, S.S. 2008. Development of Cognitive and Affective Trust in Teams: A Longitudinal Study. Small Group Research 39(6): 746-69. doi:10.1177/1046496408323569.

Wong, G., T. Greenhalgh, G. Westhorp and R. Pawson. 2014. Development of Methodological Guidance, Publication Standards and Training Materials for Realist and Meta-Narrative Reviews: The RAMESES (Realist and Meta-Narrative Evidence Syntheses-Evolving Standards) Project. Health Services Delivery Research 2: 30.

\section{$<$ H1 $>$ About the Authors}

$<$ TX1>Maura MacPhee, RN, PhD CFNEI, is a Professor of Nursing at the University of British Columbia and teaches healthcare leadership and administration. She is a health services researcher who studies work environment factors related to psychological health and well-being. She can be reached be e-mail at maura.macphee@ubc.ca or by phone at 1-604-822-2891. 
$<$ TX2> Geoffrey Wong, MA MBBS MRCGP FHEA MD(Res), is a part-time clinical research fellow at the University of Oxford and Family Physician in the United Kingdom's National Health Service. His research interests are in the use of realist research approaches to make sense of complex healthcare interventions so as to be able to produce findings that are useful to patients, clinicians, policy and decision makers. He can be reached by e-mail at geoffrey.wong@phc.ox.ac.uk or by phone at +44 1865289300.

$<$ TX2>Ruth Abrams, BA, MSc, MRes, is a researcher at University College London, London, United Kingdom. She is an organizational psychologist whose research focuses on role boundaries among homecare workers, work delegation within primary care and multidisciplinary teamwork in general healthcare settings. She can be reached by e-mail at r.abrams@ucl.ac.uk or by phone at +442077940500 .

$<$ TX2>Katelyn Merrett, RN, BA, BSN, MSN(c), is a research assistant at the University of British Columbia and a practicing registered nurse in acute renal and hospice care. 
HQ 23.2_MacPhee_Table

\section{$<T B>T A B L E$ 1. CMOCs for team processes program theory}

\begin{tabular}{|l|l|}
\hline$\#$ & $\begin{array}{l}\text { Forming: Establishing social ground rules, learning about each other's scope and roles and } \\
\text { creating a psychologically safe workplace }\end{array}$ \\
\hline 1 & $\begin{array}{l}\text { CMOC: When team members have opportunities to interact with each other (C), perspective- } \\
\text { taking occurs (M), laying the foundation for social bonds and trusting relationships (O). }\end{array}$ \\
\hline 2 & $\begin{array}{l}\text { CMOC: When teams have dedicated supports in place (C), team members have increased } \\
\text { opportunities for perspective-taking (M), resulting in more awareness of each other's roles and } \\
\text { scopes (O). }\end{array}$ \\
\hline 3 & $\begin{array}{l}\text { CMOC: When team members are aware, interested and concerned about others (C), they strive } \\
\text { to demonstrate carefulness in their actions and words (M), resulting in greater workplace } \\
\text { psychological safety (O). }\end{array}$ \\
\hline 4 & $\begin{array}{l}\text { Storming: Working out conflict and narrowing the us-them gap } \\
\text { taking (M), resulting in more conflict, less willingness to work collaboratively and a greater us- } \\
\text { them gap (O). }\end{array}$ \\
\hline 5 & $\begin{array}{l}\text { Norming: Developing deeper, affective trust and a collective team identity } \\
\text { with each other (C), because of perspective-taking (M), they begin developing affective trust in } \\
\text { one another (O). }\end{array}$ \\
\hline 6 & $\begin{array}{l}\text { CMOC: When team members openly share information and ideas (C), because of affective trust } \\
\text { (M), they develop a collective team identity (O). }\end{array}$ \\
\hline 7 & $\begin{array}{l}\text { Performing: Managing ambiguity and inviting change and innovation } \\
\text { (M), they are comfortable with ambiguity and have the capacity to champion innovation (O). }\end{array}$ \\
\hline
\end{tabular}

$<$ TB $>$ TABLE 2. Evidence-informed strategies for key team processes mechanisms

\begin{tabular}{|c|c|}
\hline Mechanism & Strategies \\
\hline Perspective-taking & $\begin{array}{l}\text { - Routinized, frequent interactions } \\
\text { - Briefing, huddles and debriefing } \\
\text { - Team meetings }\end{array}$ \\
\hline Carefulness & $\begin{array}{l}\text { Relational education } \\
\text { - Mindfulness } \\
\text { - Appreciative Inquiry } \\
\text { - Reflected best self }\end{array}$ \\
\hline Trust & $\begin{array}{l}\text { - Formal, facilitated orientation } \\
\text { - Creation of team ground rules }\end{array}$ \\
\hline
\end{tabular}


\begin{tabular}{|l|l|}
\hline & $\bullet$ Shadow experiences \\
\hline
\end{tabular} 
HQ 23.2_MacPhee_Figure

$<$ FIG $>$ FIGURE 1. Program theory for team processes in Canadian primary healthcare settings

\begin{tabular}{|llll|}
\hline Forming & Storming & Norming & Performing \\
Establishing social ground rules & Working out conflict & Co-creating new norms & Managing ambiguity \\
Learning each other's scope, roles & & Forging a collective identity & Inviting innovation \\
& Sharing critical information \\
& Sharing decisions \\
\hline & Leadership & \\
\hline
\end{tabular}

